\title{
Low frequency impedance of tapered transitions with arbitrary cross sections
}

\author{
G. Stupakov \\ Stanford Linear Accelerator Center, Stanford University, Stanford, California 94309, USA
}

(Received 30 July 2007; published 20 September 2007)

\begin{abstract}
We study the impedance of a tapered transition at small frequencies for an arbitrary shape of the transition cross section. Our approach does not require a symmetry axis in the system (unlike round geometry). We show that the calculation of the impedance reduces to finding a few auxiliary potential functions that satisfy two-dimensional Poisson equations with Dirichlet boundary conditions. In simple cases such solutions can be obtained analytically; for more complicated geometries they can easily be found numerically. We apply our method to axisymmetric geometry and reproduce results known from the literature. We then calculate the impedance of a taper with rectangular cross section in which the vertical dimension of the cross section is a slowly changing function of the longitudinal coordinate. Finally, we find a transverse kick experienced by a beam passing near a conducting wall with a variable distance from the beam to the wall.
\end{abstract}

DOI: 10.1103/PhysRevSTAB.10.094401

PACS numbers: $41.20 . \mathrm{Jb}, 41.60 .-\mathrm{m}$

\section{INTRODUCTION}

The calculation of the impedance for the elements of a vacuum chamber system and the associated calculation of beam dynamics effects, such as beam instabilities or wakefield induced emittance growth, are important elements in the design of a modern accelerator. Various discontinuities between segments of the vacuum chamber are common in practice. To avoid abrupt transitions small-angle tapers are often used to minimize the impedance of such discontinuities. For example, collimators with such tapers will be used in the postlinac collimation section of the ILC.

Computer simulations of tapers are not always easy to carry out, especially in cases when the taper cross section is strongly elongated in one direction. Several analytical approaches have been developed in the past to treat gradual tapers. Yokoya derived both the longitudinal and transverse impedance of a smooth axisymmetric transition in the lowfrequency approximation [1]. His result for the dipolar impedance of a transition that connects identical pipes is given by the following equation:

$$
Z^{\text {round }}=-\frac{i Z_{0}}{2 \pi} \int d z\left(\frac{a^{\prime}}{a}\right)^{2},
$$

where $Z_{0}=377 \mathrm{Ohm}, a$ is the radius of the pipe, and the prime denotes derivative with respect to $z$. Yokoya's method was later simplified and extended to the case of rectangular geometry [2,3]. It was also found in Ref. [3] that, in the limit of small frequencies, the impedance of a rectangular transition increases linearly with the width of the taper if the ratio of the width of the transition to the gap becomes large. The vertical impedance for a taper with rectangular cross section (after correction of an erroneous numerical factor 2 in [3], see Sec. V), is

$$
Z_{y}^{\mathrm{rect}}=-\frac{i w Z_{0}}{4} \int d z \frac{\left(g^{\prime}\right)^{2}}{g^{3}},
$$

where $w$ is the (constant) width in the $x$ direction, and $g$ is the (varying) gap of the taper in the $y$ direction; it is assumed that $g \ll w \ll l$, where $l$ is the characteristic length of the collimator. Recently, Podobedov and Krinsky found the impedance of a taper with an elliptical cross section and confirmed the same linear dependence of the transverse impedance on the ellipse elongation $g / w$ in the limit $g \ll w[4]$. The limit of very wide transitions, $w \rightarrow \infty$, of a flat collimator was studied in Ref. [5]. Note that a much more detailed theory of impedance was developed by Warnock for a shallow taper in an axisymmetric geometry, when the variation of the pipe radius $\Delta a$ is small, $\Delta a \ll a$, [6].

As was mentioned above, Eqs. (1) and (2) are valid at low frequencies. For the round taper, the requirement of low frequencies reads $\omega a^{2} / c \ll l[2]$. For highly elongated rectangular cross section taper, the applicability condition of low frequencies is more complicated, see [7].

The goal of this paper is twofold. First, we generalize the previous approaches to the problem of impedance calculation at small frequencies for an arbitrary shape of the transition cross section. An illustration of such a transition is schematically shown in Fig. 1: it does not require a symmetry axis in the system (unlike round, rectangular, or elliptic geometries studied before). It requires however, that the characteristic length of the transition $l$ is much larger than the typical transverse size $a$ :

$$
l \gg a \text {. }
$$

Our second goal is to reproduce here the result of the unpublished paper [2] for the rectangular shape of the transition (and, as was mentioned above, to correct a wrong numerical factor in the impedance).

We use the Gaussian system of units throughout this paper; to convert impedances to MKS units, one multiplies them by the factor $Z_{0} c / 4 \pi$. 


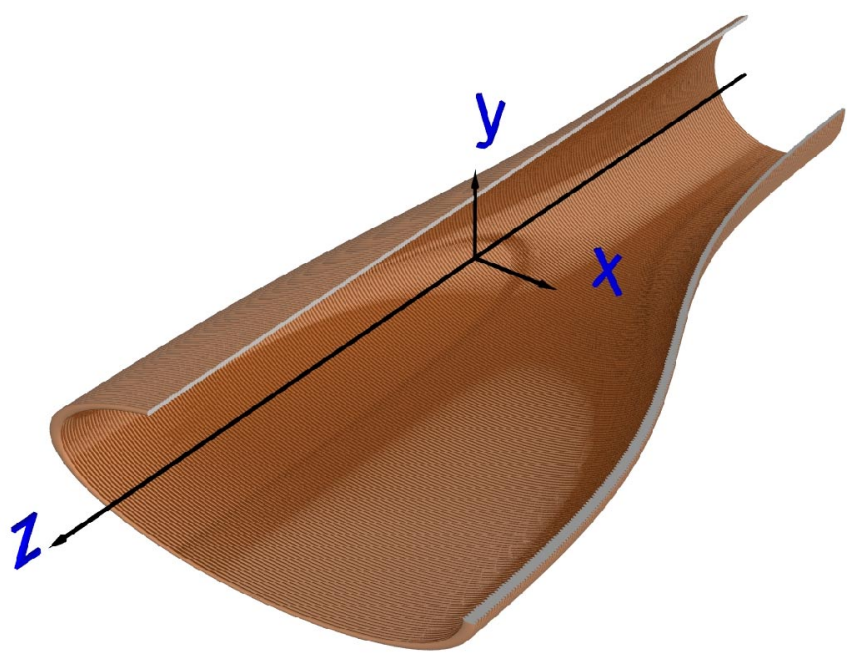

FIG. 1. (Color) A smooth transition of a beam pipe (a cutaway drawing shows the interior part of the pipe).

\section{MAIN EQUATIONS AND APPROXIMATIONS}

The geometry of the transition under study is schematically shown in Fig. 1. The transition connects two cylindrical pipes with different cross sections, with the $z$ axis going parallel to the walls of these pipes. Mathematically this means that in the limits $z \rightarrow \pm \infty$ the cross sections $z=$ const tend to some limiting curves in the $x-y$ plane (which are cross sections of the incoming and outgoing pipes). Note that the $z$ axis is not required to go through the center of the pipes. We consider the characteristic length $l$ of the transition as a large parameter; in addition, the frequency $\omega$ is considered as a small parameter. We also assume metallic boundaries with perfect conductivity.

For calculation of the impedance we consider a beam of infinitesimally small transverse size shifted from the axis of the system to a position given by coordinates $x_{1}$ and $y_{1}$. The beam propagates along the $z$ axis with the speed of light $c$. We will use the Fourier transform with respect to time and assume the harmonic time dependence of all quantities with the frequency $\omega$. The charge and current densities of the beam are given by the following equations:

$$
\rho=\tilde{\rho} e^{-i \omega t}, \quad \tilde{\rho}=\delta\left(\boldsymbol{r}-\boldsymbol{r}_{1}\right) e^{i k z}, \quad j=\hat{z} c \rho,
$$

where $k=\omega / c, \hat{z}$ is the unit vector in the direction of the $z$ axis, and we use the notation $\boldsymbol{r}$ for the two-dimensional vector $(x, y)$ in the plane transverse to the direction of propagation of the beam. The vector $\boldsymbol{r}_{1}=\left(x_{1}, y_{1}\right)$ specifies the transverse offset of the beam. We will also use a slightly different terminology, related to the calculation of the wakefields, associating vector $\boldsymbol{r}_{1}$ with the position of the leading particle, and associating vector $\boldsymbol{r}$ with the position of the trailing one.

It follows from Maxwell's equations that the electric field $\boldsymbol{E}$ satisfies the following equation:

$$
\left(\nabla^{2}+\partial_{z z}-c^{-2} \partial_{t t}\right) \boldsymbol{E}=4 \pi \nabla \rho+\frac{4 \pi}{c^{2}} \partial_{t} \boldsymbol{j}
$$

where we use the notation $\partial_{t}, \partial_{t t}, \partial_{z z}$, etc., to denote partial derivatives with respect to the variables indicated by the subscripts.

We now introduce a formal small parameter $\epsilon$ of the order of the ratio $a / l$ with $a$ being a characteristic transverse size of the transition. The electric field is represented as

$$
\boldsymbol{E}=[\boldsymbol{e}(\boldsymbol{r}, z)+\epsilon \hat{z} \mathcal{E}(\boldsymbol{r}, z)] e^{-i \omega t},
$$

with $\boldsymbol{e}$ the transverse part of the field, $\boldsymbol{e} \cdot \hat{z}=0$, and $\mathcal{E}$ the longitudinal part. In Eq. (6) we indicate that the longitudinal part of the field in a long transition is small compared to the transverse one (it would be exactly equal to zero in a pipe with constant cross section in the limit $v=c$ ). The longitudinal impedance $Z_{\|}$of the transition is given by the integral

$$
Z_{\|}\left(\boldsymbol{r} ; \boldsymbol{r}_{1}\right)=-\frac{1}{c} \int_{-\infty}^{\infty} e^{-i k z} \mathcal{E}(\boldsymbol{r}, z) d z,
$$

where in the arguments of $Z_{\|}$we indicated both the offset of the integration path given by the vector $\boldsymbol{r}$, and the offset $\boldsymbol{r}_{1}$ of the beam (to simplify notation we drop $\boldsymbol{r}_{1}$ from the arguments of $\mathcal{E}$, however).

Knowledge of the longitudinal impedance allows one to compute the transverse impedance using the PanofskyWenzel theorem [8]. In the general case, the transverse impedance is represented by vector $\boldsymbol{Z}_{\perp}$ perpendicular to the beam orbit, and is given by

$$
Z_{\perp}=\frac{c}{\omega} \nabla_{\perp} Z_{\|}
$$

where $\nabla_{\perp}$ is the transverse operator that differentiates with respect to the coordinates $\boldsymbol{r}, \nabla_{\perp}=\hat{\boldsymbol{x}} \partial_{x}+\hat{\boldsymbol{y}} \partial_{y}$, with $\hat{\boldsymbol{x}}$ and $\hat{\boldsymbol{y}}$ being the unit vectors in $x$ and $y$ directions.

In many applications it is assumed that there is a symmetry axis in the system and the beam has a small offset relative to this axis compared to the transverse size of the pipe. In this case, one can expand the impedance in Taylor series. The leading terms in the transverse impedance in this case are linear in offsets of the leading and trailing particles. ${ }^{1}$

As we will see from the result, for a transition that connects pipes of equal cross sections, the lowest order terms in the longitudinal impedance are proportional to $\omega$ (if the cross sections are different, $Z_{\|}$might have a term that does not depend on frequency). Since we assume that the frequency is small, it allows us to neglect terms $O\left(\omega^{2}\right)$

\footnotetext{
${ }^{1}$ As is well known, for axisymmetric systems the transverse impedance does not depend on the offset of the trailing particle. This however is not true for systems which are not axisymmetric (see, e.g., [9]).
} 
throughout this paper, and, in particular, to neglect the term $c^{-2} \partial_{t t}=-c^{-2} \omega^{2}$ in Eq. (5).

To account for a slow variation of fields along the $z$ axis, we will replace in this equation $\partial_{z z} \rightarrow \epsilon^{2} \partial_{z z}$, because the second derivative along the axis of the transition scales as the inverse square of the large parameter $l$ (similarly, we will replace $\partial_{z} \rightarrow \epsilon \partial_{z}$ in equations that have the first derivative along $z$ ). With these modifications, the transverse and longitudinal components of Eq. (5) are

$$
\begin{gathered}
\left(\nabla_{\perp}^{2}+\epsilon^{2} \partial_{z z}\right) \boldsymbol{e}=4 \pi \nabla_{\perp} \tilde{\rho}, \\
\left(\nabla_{\perp}^{2}+\epsilon^{2} \partial_{z z}\right) \mathcal{E}=0,
\end{gathered}
$$

where we used Eqs. (4). These two equations have to be complemented by the equation $\nabla \cdot \boldsymbol{E}=4 \pi \rho$, which, in our notation, takes the form

$$
\nabla_{\perp} \cdot \boldsymbol{e}+\epsilon^{2} \partial_{z} \mathcal{E}=4 \pi \tilde{\rho},
$$

where the second order term on the left-hand side is the result of applying the first order derivative $\epsilon \partial_{z}$ to the longitudinal field $\epsilon \mathcal{E}$.

Equations (9)-(11) are to be solved with the boundary condition of zero tangential electric field on the surface of the metal

$$
\boldsymbol{E} \times\left.\boldsymbol{n}\right|_{S}=0,
$$

where $\boldsymbol{n}$ is the unit vector normal to the surface, and the subscript $S$ indicates a position on the metallic surface. It is convenient to split this boundary condition into two components by introducing a transverse vector $\boldsymbol{m}$ perpendicular to the $z$ axis, and the longitudinal component $n_{z}$ of the vector $\boldsymbol{n}$ such that

$$
\boldsymbol{n}=\boldsymbol{m}+\boldsymbol{\epsilon} \hat{z} n_{z}
$$

with $\boldsymbol{m} \cdot \hat{z}=0$ and $m^{2}=1-\epsilon^{2} n_{z}^{2} \approx 1$. Note that the smallness of the longitudinal component of vector $\boldsymbol{n}$ is reflected in the parameter $\epsilon$ in the second term of Eq. (13). It is easy to check that, in terms of the fields $\boldsymbol{e}$ and $\mathcal{E}$, the boundary condition (12) is now split into two:

$$
\begin{gathered}
\boldsymbol{e} \times\left.\boldsymbol{m}\right|_{S}=0, \\
\left.\mathcal{E}\right|_{S}=\left.\frac{n_{z}}{m^{2}} \boldsymbol{m} \cdot \boldsymbol{e}\right|_{S}
\end{gathered}
$$

\section{PERTURBATION THEORY OF APPROXIMATE SOLUTION OF FIELD EQUATIONS}

We seek a solution of Eqs. (9)-(11) with the boundary conditions (14) and (15) as a series in the small parameter $\epsilon$ :

$$
\begin{aligned}
& \boldsymbol{e}(\boldsymbol{r}, z)=\boldsymbol{e}_{0}(\boldsymbol{r}, z)+\boldsymbol{\epsilon}^{2} \boldsymbol{e}_{2}(\boldsymbol{r}, z)+\cdots, \\
& \mathcal{E}(\boldsymbol{r}, z)=\mathcal{E}_{1}(\boldsymbol{r}, z)+\boldsymbol{\epsilon}^{2} \mathcal{E}_{3}(\boldsymbol{r}, z)+\cdots,
\end{aligned}
$$

where the subscripts indicate the smallness of the corresponding quantity [note that we start the expansion of $\mathcal{E}$ from the first order term because the physical longitudinal field is introduced in Eq. (6) as $\epsilon \mathcal{E}$ ].

Substituting Eqs. (16) into Eqs. (9) and (11), in the lowest order, we find

$$
\begin{gathered}
\nabla_{\perp}^{2} \boldsymbol{e}_{0}=4 \pi \nabla_{\perp} \tilde{\boldsymbol{\rho}}, \\
\nabla_{\perp} \cdot \boldsymbol{e}_{0}=4 \pi \tilde{\rho},
\end{gathered}
$$

with the boundary condition (14) applied to the zeroth order vector $\boldsymbol{e}_{0}$ :

$$
\boldsymbol{e}_{0} \times\left.\boldsymbol{m}\right|_{S}=0 .
$$

The differential operators in these equations act in the transverse direction, and the variable $z$ comes in as a parameter. We seek a solution to these equations in the form of the transverse potential field $\phi_{0}(\boldsymbol{r}, z)$ :

$$
\boldsymbol{e}_{0}=-e^{i k z} \nabla_{\perp} \phi_{0}(\boldsymbol{r}, z) .
$$

Substituting this expression into Eq. (18) yields

$$
\nabla_{\perp}^{2} \phi_{0}=-4 \pi \delta\left(\boldsymbol{r}-\boldsymbol{r}_{1}\right) .
$$

It is easy to see that, if this equation is satisfied, then Eq. (17) is satisfied too. The boundary condition for the potential $\phi_{0}$ follows from Eq. (19):

$$
\left.\phi_{0}\right|_{S}=0 .
$$

We see that $\phi_{0}$ is the Green function for the twodimensional Poisson equation with the Dirichlet boundary condition in a given cross section of the transition at coordinate $z$. A general expression for such Green's functions is known for several typical geometries, e.g., round, rectangular, elliptical, etc. It can also be relatively easily computed numerically for a given shape of the cross section.

We now use Eq. (10) to the lowest order:

$$
\nabla_{\perp}^{2} \mathcal{E}_{1}=0 .
$$

The boundary condition for this equation follows from Eqs. (15) and (20):

$$
\left.\mathcal{E}_{1}\right|_{S}=-\left.e^{i k z} \frac{n_{z}}{m^{2}} \boldsymbol{m} \cdot \nabla_{\perp} \phi_{0}\right|_{S} .
$$

We will now show that the solution to Eq. (23) with the above boundary condition is given by

$$
\mathcal{E}_{1}=-e^{i k z} \partial_{z} \phi_{0} .
$$

Indeed, because $\phi_{0}$ satisfies Eq. (21), we have $\partial_{z} \nabla_{\perp}^{2} \phi_{0}=$ 0 which means that $\mathcal{E}_{1}$ given by Eq. (25) satisfies Eq. (23). Also, since $\phi_{0}=0$ at the boundary, the gradient of $\phi_{0}$ on $S$ directed along the normal vector $\boldsymbol{n}$

$$
\left.\nabla \phi_{0}\right|_{S}=\boldsymbol{n} R(\boldsymbol{r}, z),
$$


where $R$ is a scalar function. Projecting this equation onto the vectors $\boldsymbol{m}$ and $\hat{z}$, it is easy to show that

$$
\left.\frac{\partial \phi_{0}}{\partial z}\right|_{S}=\left.\frac{n_{z}}{m^{2}} \boldsymbol{m} \cdot \nabla_{\perp} \phi_{0}\right|_{S},
$$

which is equivalent to the boundary condition Eq. (24).

The solution given by Eqs. (20) and (25) shows that the combination of the zeroth order transverse and the first order longitudinal fields is represented by the potential function $\phi_{0}$. The contribution to the impedance of the longitudinal component of the electric field given by (25) is

$$
\begin{aligned}
Z_{\|}^{(1)}\left(\boldsymbol{r} ; \boldsymbol{r}_{1}\right) & =-\frac{1}{c} \int_{-\infty}^{\infty} e^{-i k z} \mathcal{E}_{1}(\boldsymbol{r}, z) d z=\frac{1}{c} \int_{-\infty}^{\infty} \partial_{z} \phi_{0} \\
& =\frac{1}{c}\left[\phi_{0}(z \rightarrow \infty)-\phi_{0}(z \rightarrow-\infty)\right] .
\end{aligned}
$$

This contribution vanishes if the incoming and outgoing pipes connected to the transition are identical. In what follows, we calculate the next order of the perturbation theory which gives a nonzero result for the case of identical pipes. (11):

We first select terms of the second order in Eqs. (9) and

$$
\begin{aligned}
& \nabla_{\perp}^{2} \boldsymbol{e}_{2}+\partial_{z z} \boldsymbol{e}_{0}=0, \\
& \nabla_{\perp} \cdot \boldsymbol{e}_{2}+\partial_{z} \mathcal{E}_{1}=0 .
\end{aligned}
$$

The boundary condition is obtained by taking the second order terms in Eq. (14):

$$
\boldsymbol{e}_{2} \times\left.\boldsymbol{m}\right|_{S}=0 .
$$

We now seek a solution as a sum of potential and solenoidal transverse fields represented, respectively, by the functions $\phi_{2}$ and $k \psi_{2}$ :

$$
\boldsymbol{e}_{2}=\left(-\nabla_{\perp} \phi_{2}+k \hat{z} \times \nabla_{\perp} \psi_{2}\right) e^{i k z},
$$

with the boundary conditions that follow from Eq. (31),

$$
\left.\phi_{2}\right|_{S}=0,\left.\quad \boldsymbol{m} \cdot \nabla_{\perp} \psi_{2}\right|_{S}=0 .
$$

Upon substitution of Eqs. (25) and (32) into Eq. (30) we obtain the following equation for $\phi_{2}$ :

$$
\nabla_{\perp}^{2} \phi_{2}=-i k \partial_{z} \phi_{0}-\partial_{z z} \phi_{0} .
$$

We then substitute Eqs. (20) and (32) into Eq. (29) and obtain the equation for $\psi_{2}$ :

$$
\nabla_{\perp}^{2}\left(\hat{z} \times \nabla_{\perp} \psi_{2}\right)=i \nabla_{\perp} \partial_{z} \phi_{0}
$$

where we neglected the second order terms in frequency on the right-hand side.

Note that if one can find a function $\psi_{0}$ such that

$$
\nabla_{\perp} \psi_{0} \times \hat{z}=\nabla_{\perp} \phi_{0}
$$

then Eq. (35) for $\psi_{2}$ can be written as

$$
\nabla_{\perp}^{2} \psi_{2}=-i \partial_{z} \psi_{0}
$$

Note that, as it follows from Eqs. (21) and (36), the curl of the two-dimensional vector field $\nabla_{\perp} \psi_{0}$ has a deltafunction singularity at $\boldsymbol{r}_{1}$. In some cases, $\psi_{0}$ can be found if one considers the sum $\phi_{0}+i \psi_{0}$ as a complex function of the complex variable $\zeta=x+i y$. The function $\phi_{0}+i \psi_{0}$ is analytical in the region bounded by $S$ everywhere except for the point $\zeta_{1}=x_{1}+i y_{1}$. Given $\phi_{0}$, finding $\psi_{0}$ is equivalent to the finding of the imaginary part of an analytical function with a given real part.

We now come back to Eq. (34) and split $\phi_{2}$ into the sum $\phi_{2}^{(1)}+k \phi_{2}^{(2)}$ of two terms one of which does not depend on frequency, and the other one is linear in $k$,

$$
\begin{aligned}
& \nabla_{\perp}^{2} \phi_{2}^{(1)}=-\partial_{z z} \phi_{0}, \\
& \nabla_{\perp}^{2} \phi_{2}^{(2)}=-i \partial_{z} \phi_{0},
\end{aligned}
$$

with the boundary conditions $\left.\phi_{2}^{(1)}\right|_{S}=\left.\phi_{2}^{(2)}\right|_{S}=0$. Again, finding $\phi_{2}$ and $\psi_{2}$ reduces to the two-dimensional Poisson equation with the Dirichlet boundary conditions, and we assume that this problem can be solved, either analytically or numerically.

The last step in the derivation is to substitute Eqs. (16) into Eq. (10) and to select the third order terms,

$$
\nabla_{\perp}^{2} \mathcal{E}_{3}=-\partial_{z z} \mathcal{E}_{1} \approx e^{i k z} \partial_{z z z} \phi_{0}+2 i k e^{i k z} \partial_{z z} \phi_{0},
$$

with the boundary condition that follows from Eq. (15) in the third order:

$$
\begin{aligned}
\left.\mathcal{E}_{3}\right|_{S} & \left.\approx n_{z} \boldsymbol{m} \cdot \boldsymbol{e}_{2}\right|_{S} \\
& =n_{z}\left(-\boldsymbol{m} \cdot \nabla_{\perp} \phi_{2}+k(\boldsymbol{m} \times \hat{z}) \cdot \nabla_{\perp} \psi_{2}\right) e^{i k z} .
\end{aligned}
$$

The equation for $\mathcal{E}_{3}$ can be simplified by introducing a new function $\tilde{\mathcal{E}}$ related to $\mathcal{E}_{3}$ through the following equation:

$$
\mathcal{E}_{3}=e^{i k z}\left(-\partial_{z} \phi_{2}^{(1)}-2 k \partial_{z} \phi_{2}^{(2)}+k \tilde{\mathcal{E}}\right) .
$$

Using Eq. (39), one can show that $\tilde{\mathcal{E}}$ satisfies the Laplace equation,

$$
\nabla_{\perp} \tilde{\mathcal{E}}=0
$$

with the boundary condition that follows from Eq. (41):

$$
\left.\tilde{\mathcal{E}}\right|_{S}=n_{z}\left(-\boldsymbol{m} \cdot \nabla_{\perp} \phi_{2}^{(2)}+(\boldsymbol{m} \times \hat{z}) \cdot \nabla_{\perp} \psi_{2}\right) .
$$

Substituting Eq. (42) into Eq. (7) we find that the impedance is

$$
Z_{\|}=Z_{\|}^{(1)}+Z_{\|}^{(3)},
$$

with $Z_{\|}^{(1)}$ given by Eq. (28) and

$$
Z_{\|}^{(3)}\left(\boldsymbol{r} ; \boldsymbol{r}_{1}\right)=-\frac{k}{c} \int_{-\infty}^{\infty} \tilde{\mathcal{E}}(\boldsymbol{r}, z) d z
$$


Summarizing this section, we emphasize that our final result, Eqs. (28) and (46) for the impedance, is formulated in terms of a few linked solutions of the two-dimensional Poisson equations. The third order contribution, $Z_{\|}^{(3)}$, is explicitly proportional to the frequency $\omega$. In the next section we will apply the technique developed above to the case of the round geometry to show that it reproduces Yokoya's results. In Sec. V we apply this technique to the case of rectangular geometry.

\section{AXISYMMETRIC TRANSITIONS}

We now apply the above derived equations for a special case of an axisymmetric transition shown in Fig. 2. The transition is characterized by the pipe radius $a(z)$ varying along the $z$ axis.

It is known from the complex analysis, that in this case both functions $\phi_{0}$ and $\psi_{0}$ are given by the real and imaginary parts of the following expression:

$$
\phi_{0}+i \psi_{0}=-2 \ln \frac{a\left(\zeta-\zeta_{1}\right)}{a^{2}-\zeta \zeta_{1}},
$$

where $\zeta=x+i y$ and $\zeta_{1}=x_{1}+i y_{1}$. In what follows we will use the cylindrical coordinates $r=|\zeta|$ and $\theta=$ $\arg (\zeta)$.

We first derive the longitudinal impedance for the case when the leading particle is moving along the $z$ axis, $\zeta_{1}=$ 0 . We have

$$
\phi_{0}=-2 \ln \frac{r}{a}, \quad \psi_{0}=-2 \arg (\zeta)=-2 \theta .
$$

The function $\phi_{2}^{(2)}$ depends only on radius $r$, and Eq. (39) for $\phi_{2}^{(2)}$ reads

$$
\nabla^{2} \phi_{2}^{(2)}=-i \partial_{z} \phi_{0}=-2 i \frac{a^{\prime}}{a},
$$

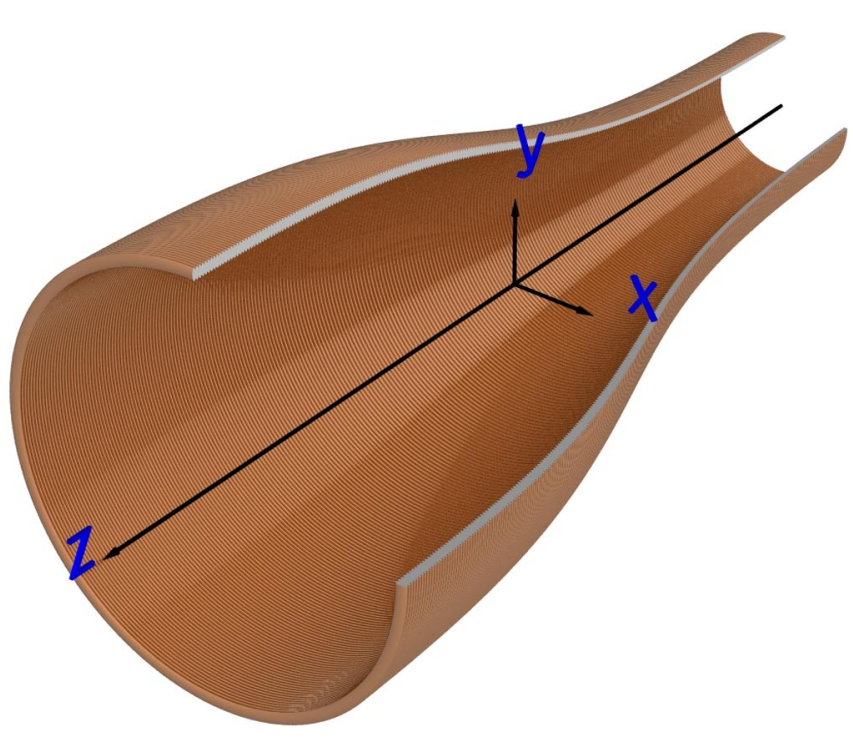

FIG. 2. (Color) Geometry of a round transition. where the prime denotes the derivative with respect to $z$. From this equation and the boundary condition $\left.\phi_{2}^{(2)}\right|_{r=a}=$ 0 , we find

$$
\phi_{2}^{(2)}=-\frac{1}{2} i \frac{a^{\prime}}{a}\left(r^{2}-a^{2}\right)
$$

For the function $\psi_{2}$ we have from Eq. (37) $\nabla_{\perp}^{2} \psi_{2}=0$, and, with the boundary condition in Eq. (33), it follows that $\psi_{2}=0$.

We now need to solve the equation for $\tilde{\mathcal{E}}(r)$ :

$$
\frac{1}{r} \frac{\partial}{\partial r} r \frac{\partial \tilde{\mathcal{E}}}{\partial r}=0
$$

with the boundary condition

$$
\left.\tilde{\mathcal{E}}\right|_{r=a}=-a^{\prime} \frac{\partial \phi_{2}^{(2)}}{\partial r}=i\left(a^{\prime}\right)^{2}
$$

The solution is a constant value $\tilde{\mathcal{E}}=i\left(a^{\prime}\right)^{2}$.

Using Eqs. (28) and (46), we find the well-known result for the longitudinal impedance,

$$
Z=\frac{2}{c} \ln \frac{a_{1}}{a_{2}}-i \frac{k}{c} \int d z\left(a^{\prime}\right)^{2}
$$

where $a_{1}=a(z \rightarrow \infty)$ and $a_{2}=a(z \rightarrow-\infty)$. In the case of identical pipes, $a_{1}=a_{2}$, the first term in this equation vanishes, and the impedance becomes purely inductive.

We will now calculate the transverse impedance in the axisymmetric case, assuming a small offset of the leading particle in the direction of the $x$ axis, ${ }^{2} \zeta_{1}=x_{1} \ll a$, and linearizing equations in the small parameter $x_{1}$. As it follows from Eq. (47), the linear in $x_{1}$ parts of $\phi_{0}$ and $\psi_{0}$ are

$$
\begin{aligned}
\phi_{0} & =-2 x_{1} \cos \theta\left(\frac{r}{a^{2}}-\frac{1}{r}\right), \\
\psi_{0} & =-2 x_{1} \sin \theta\left(\frac{r}{a^{2}}+\frac{1}{r}\right) .
\end{aligned}
$$

This gives the following equation for $\phi_{2}^{(2)}$ (for brevity of notation, we drop the superscript $d$ in subsequent functions $\phi$ and $\psi$ ):

$$
\frac{1}{r} \frac{\partial}{\partial r} r \frac{\partial \phi_{2}^{(2)}}{\partial r}+\frac{1}{r^{2}} \frac{\partial^{2} \phi_{2}^{(2)}}{\partial r^{2}}=-4 i \frac{r a^{\prime}}{a^{3}} \cos \theta
$$

With the boundary condition $\left.\phi_{2}^{(2)}\right|_{r=a}=0$, the solution is

\footnotetext{
${ }^{2} \mathrm{An}$ offset in the arbitrary direction can always be oriented along the $x$ axis by a rotation of the coordinates.
} 


$$
\phi_{2}^{(2)}=-x_{1} \frac{i a^{\prime}}{2 a^{3}}\left(r^{3}-r a^{2}\right) \cos \theta .
$$

Analogously, the equation for $\psi_{2}$ reads

$$
\frac{1}{r} \frac{\partial}{\partial r} r \frac{\partial \psi_{2}}{\partial r}+\frac{1}{r^{2}} \frac{\partial^{2} \psi_{2}}{\partial r^{2}}=-4 i \frac{r a^{\prime}}{a^{3}} \sin \theta
$$

and with the boundary condition $\left.\partial_{r} \psi_{2}\right|_{r=a}=0$ the solution is

$$
\psi_{2}=-\frac{1}{2} x_{1} i \frac{a^{\prime}}{a^{3}}\left(r^{3}-3 r a^{2}\right) \sin \theta .
$$

With the given functions $\phi_{2}^{(2)}$ and $\psi_{2}$, we can formulate the boundary condition Eq. (44) for $\tilde{\mathcal{E}}$ :

$$
\left.\tilde{\mathcal{E}}\right|_{r=a}=2 i x_{1} \frac{\left(a^{\prime}\right)^{2}}{a} \cos \theta,
$$

and solving Eq. (43) we find

$$
\tilde{\mathcal{E}}=2 i x_{1} \frac{r\left(a^{\prime}\right)^{2}}{a} \cos \theta=2 i x_{1} x \frac{\left(a^{\prime}\right)^{2}}{a} .
$$

The total dipole longitudinal impedance (45) can now be calculated (where the superscript $d$ stands for the "dipole" component):

$$
Z_{\|}^{d}=\frac{2 x_{1} x}{c}\left(\frac{1}{a_{1}^{2}}-\frac{1}{a_{2}^{2}}\right)-2 \frac{i k}{c} x x_{1} \int d z \frac{\left(a^{\prime}\right)^{2}}{a^{2}} .
$$

Using the Panofsky-Wenzel relation (8), we now find the transverse impedance of an axisymmetric transition:

$$
Z_{\perp}^{d}=\frac{1}{k} \frac{\partial Z_{\|}^{d}}{\partial x}=\frac{2 x_{1}}{\omega}\left(\frac{1}{a_{1}^{2}}-\frac{1}{a_{2}^{2}}\right)-2 \frac{i x_{1}}{c} \int d z \frac{\left(a^{\prime}\right)^{2}}{a^{2}} .
$$

In the limit of equal radii $a_{1}=a_{2}$, only the second term on the right side of this equation survives. Normalized by the offset $x_{1}$ it agrees with Yokoya's result Eq. (1).

\section{RECTANGULAR TRANSITIONS}

We will now turn our attention to the case of a rectangular transition. The geometry is shown in Fig. 3; the width of the rectangular area along $x$ is equal to $w$, and the height in the $y$ direction is equal to $g$. We assume that $g(z)$ varies along $z ; w$ however is constant. It is convenient to choose the local coordinate system for a given $z$ as shown in Fig. 3 where the origin is located at the center of the rectangle.
The beam, traveling in the $z$ direction, has an offset $\boldsymbol{r}_{1}=$ $\left(x_{1}, y_{1}\right)$.

The first step in calculation is to find the Green function $\phi_{0}$ for a rectangular domain that satisfies Eq. (21) with the boundary condition (22). There exist several representations of such Green function in the literature. We found that the most convenient one for the purposes of this study is given by the following expression ${ }^{3}[10]$ :

$$
\begin{aligned}
\phi_{0}= & 8 \sum_{n=1}^{\infty} \frac{1}{n \sinh \frac{n \pi g}{w}} \sinh \frac{n \pi\left(g / 2-y_{1}\right)}{w} \sinh \frac{n \pi(y+g / 2)}{w} \\
& \times \sin \frac{n \pi\left(x_{1}+w / 2\right)}{w} \sin \frac{n \pi(x+w / 2)}{w}, \quad y<y_{1}, \\
\phi_{0}= & 8 \sum_{n=1}^{\infty} \frac{1}{n \sinh \frac{n \pi g}{w}} \sinh \frac{n \pi(g / 2-y)}{w} \sinh \frac{n \pi\left(y_{1}+g / 2\right)}{w} \\
& \times \sin \frac{n \pi\left(x_{1}+w / 2\right)}{w} \sin \frac{n \pi(x+w / 2)}{w}, \quad y>y_{1} .
\end{aligned}
$$

Using this expression for $\phi_{0}$, one can consecutively find $\phi_{2}^{(2)}, \psi_{2}$, and $\tilde{\mathcal{E}}$ solving the corresponding equations by Fourier expansion in $x$. These calculations can be simplified if one notices that it is only the derivative $\partial_{x} \psi_{2}$, and not the function $\psi_{2}$ itself, that is required to find $\tilde{\mathcal{E}}$. Indeed, in the boundary condition (44), vector $n_{z}$ is equal to $-g^{\prime} / 2$ at the upper and lower walls, $y= \pm g / 2$, and is equal to zero at the lateral walls, $x= \pm w / 2$. Vector $\boldsymbol{m}$ is approximately equal to $(0,1)$ at $y=g / 2$, and $\boldsymbol{m}=(0,-1)$ at $y=-g / 2$. This casts the boundary condition (44) into

$$
\begin{gathered}
\left.\tilde{\mathcal{E}}\right|_{y=-g / 2}=\left.\frac{1}{2} g^{\prime}\left(\frac{\partial \phi_{2}^{(2)}}{\partial y}+\frac{\partial \psi_{2}}{\partial x}\right)\right|_{y=-g / 2}, \\
\left.\tilde{\mathcal{E}}\right|_{y=g / 2}=-\left.\frac{1}{2} g^{\prime}\left(\frac{\partial \phi_{2}^{(2)}}{\partial y}+\frac{\partial \psi_{2}}{\partial x}\right)\right|_{y=g / 2},
\end{gathered}
$$

and we see that only $\partial_{x} \psi_{2}$ is involved. One can try to find this derivative without finding the function $\psi_{2}$ itself. Differentiating Eq. (37) with respect to $x$, we obtain

$$
\nabla_{\perp}^{2} \partial_{x} \psi_{2}=-i \partial_{z} \partial_{x} \psi_{0}=i \partial_{z} \partial_{y} \phi_{0}
$$

where in the last transformation on the right side we used the equality $\partial_{x} \psi_{0}=-\partial_{y} \phi_{0}$ that follows from Eq. (36). The boundary conditions for the function $\partial_{x} \psi_{2}$ follow from Eq. (33):

$$
\begin{gathered}
\partial_{x} \psi_{2}=0 \quad \text { at } x= \pm w / 2, \\
\partial_{y}\left(\partial_{x} \psi_{2}\right)=0 \quad \text { at } y= \pm g / 2 .
\end{gathered}
$$

Skipping the lengthy calculations we present here the final result for $\tilde{\mathcal{E}}$ :

\footnotetext{
${ }^{3}$ Equation (5.4) in Ref. [10] has a typographical error that is corrected in Eq. (63).
} 

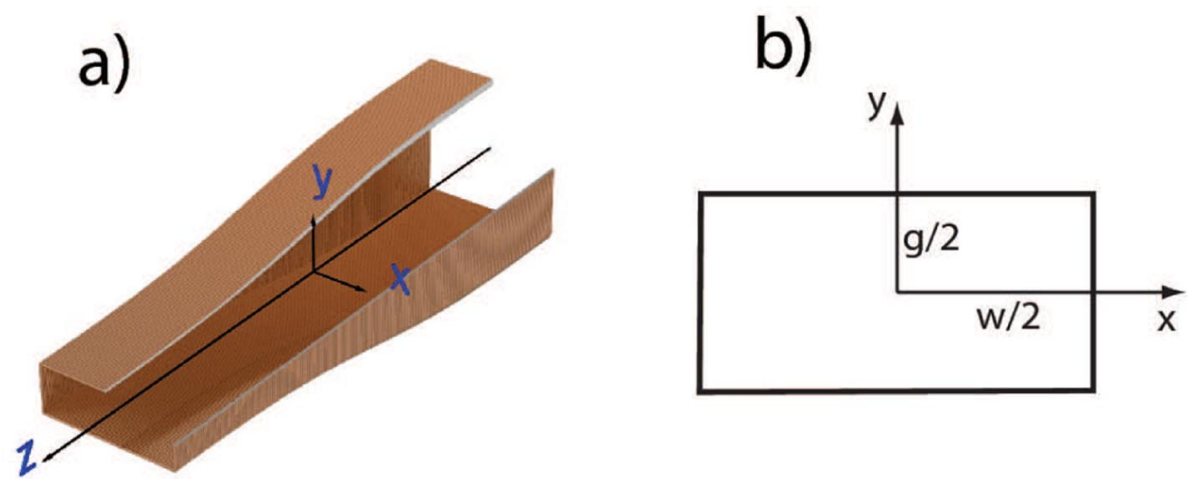

FIG. 3. (Color) A smooth transition with rectangular cross section, (a); the origin of the coordinates $(x, y)$ is located at the center of the rectangle, (b).

$$
\begin{aligned}
\tilde{\mathcal{E}}= & i\left(g^{\prime}\right)^{2} \sum_{n=1}^{\infty} \frac{1}{n \sinh ^{3} \frac{n \pi g}{w}} \sin \frac{n \pi\left(x_{1}+w / 2\right)}{w} \sin \frac{n \pi(x+w / 2)}{w} \\
& \times\left[\sinh \frac{n \pi(g / 2-y)}{w}\left(\sinh \frac{n \pi\left(3 g / 2-y_{1}\right)}{w}-3 \sinh \frac{n \pi\left(y_{1}+g / 2\right)}{w}\right)\right. \\
& \left.+\sinh \frac{n \pi(y+g / 2)}{w}\left(\sinh \frac{n \pi\left(3 g / 2+y_{1}\right)}{w}-3 \sinh \frac{n \pi\left(g / 2-y_{1}\right)}{w}\right)\right] .
\end{aligned}
$$

Equations (63) and (67) allow one to calculate the impedance for arbitrary offsets of the leading and trailing particles by computing the integrals (28) and (46). In what follows we will focus on the case when the height of the incoming and outgoing pipes far from the transition region are equal, and the first order impedance $Z_{\|}^{(1)}$ vanishes.

We first calculate the longitudinal impedance when both the leading and trailing particles are on the axis, $x=x_{1}=$ $y=y_{1}=0$. We then have

$$
Z_{\|}^{(3)}=-\frac{i k}{c} \int_{-\infty}^{\infty} d z\left(g^{\prime}\right)^{2} F\left(\frac{g}{w}\right)
$$

where

$$
\begin{aligned}
F(x)= & \sum_{m=0}^{\infty} \frac{1}{2 m+1} \operatorname{sech}^{2}\left(\frac{(2 m+1) \pi x}{2}\right) \\
& \times \tanh \left(\frac{(2 m+1) \pi x}{2}\right) .
\end{aligned}
$$

The plot of function $F(x)$ is shown in Fig. 4. In the limit $g \ll w$ corresponding to $x \ll 1$, the summation in Eq. (69) can be replaced by integration, and we have approximately $F(x) \approx 7 \zeta(3) / 2 \pi^{2}=0.43$, where $\zeta(3)$ is the Riemann zeta function. In this limit the longitudinal impedance is

$$
Z_{\|}^{(3)}=-\frac{7 \zeta(3)}{2 \pi^{2}} \frac{i k}{c} \int_{-\infty}^{\infty} d z\left(g^{\prime}\right)^{2}
$$

In the opposite limit, $g \gg w$, the function $F$ becomes small and the impedance tends to zero. In this limit the beam field lines are closed to the vertical walls and do not "feel" the variation of the gap $g$ as the beam travels along the transition.

We will now focus on the transverse impedance assuming that all offsets are small, $x, x_{1} \ll w$ and $y, y_{1} \ll g$. Then the transverse vertical and the transverse horizontal impedances (with respect to the reference trajectory) can be written as

$$
Z_{y}=y_{1} Z_{y, d}+y Z_{y, q}, \quad Z_{x}=x_{1} Z_{x, d}+x Z_{x, q},
$$

where the subscript $d$ and the subscript $q$ denote the dipole and the quadrupole contributions to the impedance, respectively. From the Panofsky-Wenzel relation (8), we find that

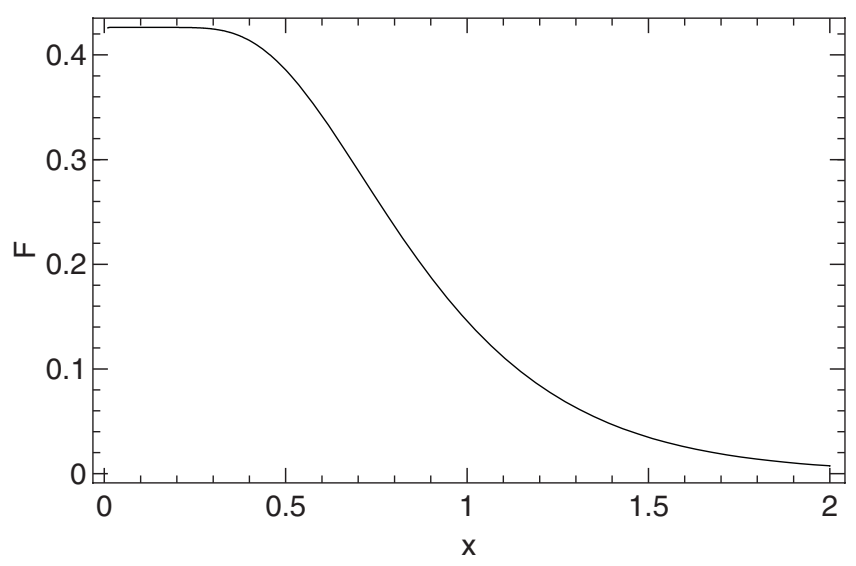

FIG. 4. Plot of function $F(x)$. 


$$
\begin{array}{ll}
Z_{y, d}=\frac{c}{\omega} \frac{\partial^{2} Z_{\|}}{\partial y \partial y_{1}}, & Z_{y, q}=\frac{c}{\omega} \frac{\partial^{2} Z_{\|}}{\partial y^{2}}, \\
Z_{x, d}=\frac{c}{\omega} \frac{\partial^{2} Z_{\|}}{\partial x \partial x_{1}}, & Z_{x, q}=\frac{c}{\omega} \frac{\partial^{2} Z_{\|}}{\partial x^{2}},
\end{array}
$$

where the derivatives on the right side are evaluated at the origin of the coordinate system. Setting $y_{1}=y_{2}=y_{0}$ and $x_{1}=x_{2}=x_{0}$, we can also define normalized total impedances:

$$
Z_{\perp, y}=\frac{Z_{y}}{y_{0}}=Z_{y, d}+Z_{y, q}, \quad Z_{\perp, x}=\frac{Z_{x}}{x_{0}}=Z_{x, d}+Z_{x, q} .
$$

With the general expression for the longitudinal impedance, all the derivatives in Eq. (72) can be easily calculated and give the following result:

$$
\begin{aligned}
Z_{y, d}^{(3)} & =-\frac{i \pi^{2} w}{c} \int_{-\infty}^{\infty} d z \frac{\left(g^{\prime}\right)^{2}}{g^{3}} G_{1}\left(\frac{g}{w}\right) \\
Z_{y, q}^{(3)} & =-Z_{x, q}^{(3)}=-\frac{i \pi^{2}}{c} \int_{-\infty}^{\infty} d z \frac{\left(g^{\prime}\right)^{2}}{g^{2}} G_{2}\left(\frac{g}{w}\right) \\
Z_{x, d}^{(3)} & =-\frac{i \pi^{2}}{c} \int_{-\infty}^{\infty} d z \frac{\left(g^{\prime}\right)^{2}}{g^{2}} G_{3}\left(\frac{g}{w}\right)
\end{aligned}
$$

where

$$
\begin{aligned}
G_{1}(x)= & x^{3} \sum_{m=0}^{\infty}(2 m+1) \\
& \times \operatorname{coth}\left(\frac{(2 m+1) \pi x}{2}\right) \operatorname{csch}^{2}\left(\frac{(2 m+1) \pi x}{2}\right) \\
G_{2}(x)= & x^{2} \sum_{m=0}^{\infty}(2 m+1) \operatorname{sech}^{2}\left(\frac{(2 m+1) \pi x}{2}\right) \\
& \times \tanh \left(\frac{(2 m+1) \pi x}{2}\right) \\
G_{3}(x)= & x^{2} \sum_{m=1}^{\infty} 2 m \operatorname{sech}^{2}(m \pi x) \tanh (m \pi x) .
\end{aligned}
$$

The plot of functions $G_{1}, G_{2}$, and $G_{3}$ is shown in Fig. 5 . Again, in the limit of small ratio $g / w$ we find

$$
\begin{aligned}
& Z_{y, d}^{(3)}=-\frac{i \pi w}{c} \int_{-\infty}^{\infty} \frac{\left(g^{\prime}\right)^{2}}{g^{3}} d z \\
& Z_{x, d}^{(3)}=Z_{y, q}^{(3)}=-Z_{x, q}^{(3)}=-\frac{i}{c} \int_{-\infty}^{\infty} d z \frac{\left(g^{\prime}\right)^{2}}{g^{2}} .
\end{aligned}
$$

The proportionality of the vertical impedance to the width of the transition in this limit was found in Ref. [2]; however, the numerical factor in the first of Eqs. (76) in that paper was erroneously found to be 2 times larger.

Note that in the limit of a very wide transition, $w \rightarrow \infty$, we have $Z_{x, d}^{(3)}=-Z_{x, q}^{(3)}$, and the total impedance in the horizontal plane becomes $Z_{x}=Z_{x, d}\left(x-x_{1}\right)$. This is an

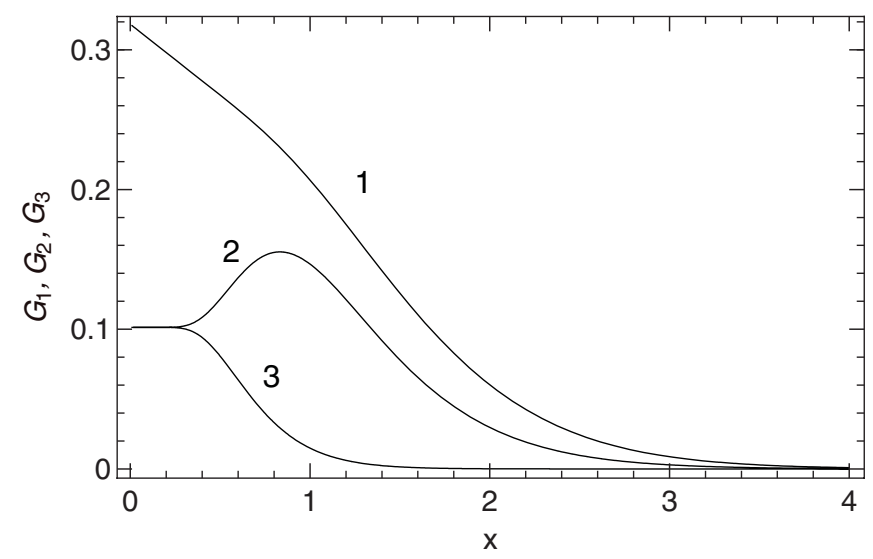

FIG. 5. Plot of functions $G_{1}(1), G_{2}(2)$, and $G_{3}$ (3).

expected result, because in this limit the vertical boundaries are removed, and the system becomes translationally invariant in the $x$ direction. ${ }^{4}$

\section{BEAM PASSING NEAR A WALL}

The two previous sections dealt with the cases when the beam was located on or near the symmetry axis of the system. In this section, to demonstrate the capabilities of our method, we consider a system that does not have a symmetry at all - a beam passing near a wall which is bent in such a way that the distance from the beam to the wall is varying in the longitudinal direction. More specifically, we assume geometry shown in Fig. 6. The wall infinitely extends in the $x$ direction, and the distance from the beam to the wall $d(z)$ in the $y$ direction varies along $z$ with $d$ approaching limiting values $d_{1}$ and $d_{2}$ at $z= \pm \infty$.

The simplest way to approach this problem is to notice that its solution can be obtained as a limit of the rectangular transition considered in the previous section, when the width $w$ and the height $g$ of the rectangle tend to infinity, but the distance from the beam to the upper wall $g(z) / 2-$ $y_{1}$ and the distance from the observation point to the wall $g(z) / 2-y$ remain finite. To discard the contribution (28) from the first order terms, we assume that the incoming and outgoing pipes have identical cross section so that this term vanishes. This means that the distances to the wall $d_{1}$ and $d_{2}$ at $z= \pm \infty$ (far from the transition) are equal.

To calculate the transverse impedance $Z_{y}$ for this problem we use the Panofsky-Wenzel relation (8) with the definition Eq. (46),

$$
Z_{y}=-\frac{1}{c} \int_{-\infty}^{\infty} \frac{\partial \tilde{\mathcal{E}}}{\partial y} d z
$$

where $\tilde{\mathcal{E}}$ is given by Eq. (67). This transverse impedance describes a kick to the beam in the $y$ direction when the

\footnotetext{
${ }^{4}$ The importance for a solution to satisfy this requirement was pointed out to the author by Krinsky.
} 


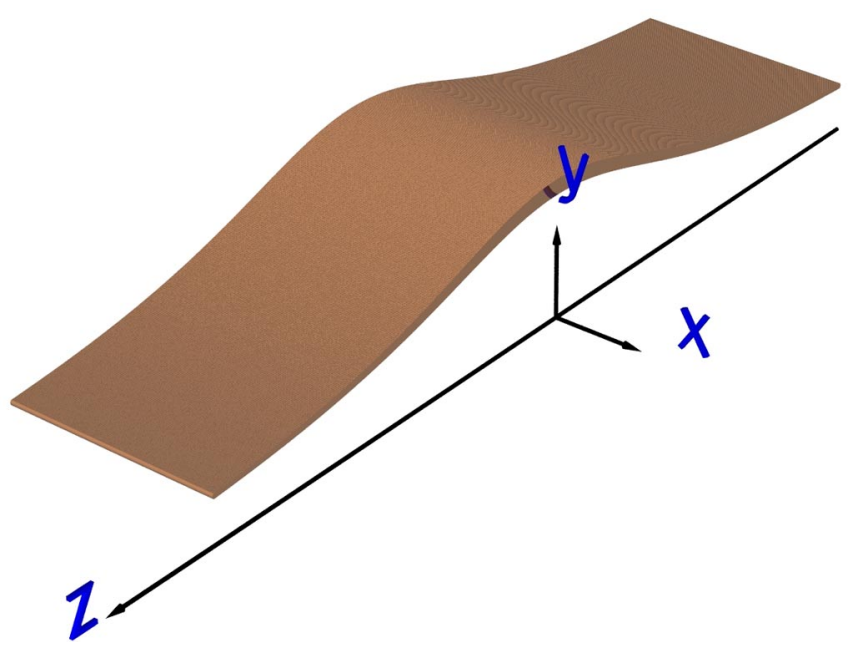

FIG. 6. (Color) A beam trajectory near a wall. Only half of the wall extending into the region of negative values of $x$ is shown.

beam propagates close to the wall. To compute the impedance on the beam trajectory, we calculate the derivative $\partial \tilde{\mathcal{E}} / \partial y$ and substitute $y=y_{1}=g / 2-d, x=x_{1}=0$, and $g^{\prime}=d^{\prime}$ into the equation:

$$
\begin{aligned}
\frac{\partial \tilde{\mathcal{E}}}{\partial y}= & \frac{2 \pi i\left(d^{\prime}\right)^{2}}{w} \sum_{n=1}^{\infty} \frac{\sin ^{2}\left(\frac{n \pi}{2}\right)}{\sinh ^{3}\left(\frac{g n \pi}{w}\right)} \\
& \times\left[\operatorname { c o s h } ( \frac { d n \pi } { w } ) \left(3 \sinh \left(\frac{(g-d) n \pi}{w}\right)\right.\right. \\
& \left.-\sinh \left(\frac{(d+g) n \pi}{w}\right)\right) \\
& +\cosh \left(\frac{(g-d) n \pi}{w}\right) \\
& \left.\times\left(\sinh \left(\frac{(2 g-d) n \pi}{w}\right)-3 \sinh \left(\frac{d n \pi}{w}\right)\right)\right] .
\end{aligned}
$$

Analysis shows that, when $d / w \rightarrow 0$, the main contribution to the sum comes from the terms with large values of $n$, and one can approximate $\sinh x, \cosh x \approx e^{x} / 2$ in Eq. (78). Since the factor $\sin ^{2}\left(\frac{n \pi}{2}\right)$ filters only odd values of $n$, summation over $n$ can be approximately replaced by integration, $\sum_{n=1}^{\infty} \sin ^{2}\left(\frac{n \pi}{2}\right) \rightarrow \frac{1}{2} \int_{0}^{\infty} d n$. This gives the following expression for $\partial \tilde{\mathcal{E}} / \partial y$ :

$$
\frac{\partial \tilde{\mathcal{E}}}{\partial y} \approx \frac{2 \pi i\left(d^{\prime}\right)^{2}}{w} \int_{0}^{\infty} e^{-2 d \pi n / w} d n=\frac{i\left(d^{\prime}\right)^{2}}{d} .
$$

Using Eq. (77) for the $y$ impedance we now find

$$
Z_{y}=-\frac{i}{c} \int_{-\infty}^{\infty} \frac{\left(d^{\prime}\right)^{2}}{d} d z
$$

We remind the reader that this result assumes that $d(z=$ $-\infty)=d(z=\infty)$.

As it follows from the symmetry consideration, for $x=$ $x_{1}$, the horizontal impedance near the wall, $Z_{x}$, vanishes.

\section{DISCUSSION}

In our solution for the rectangular transition we assumed that the width $w$ of the collimator is constant. This assumption can be easily dropped. As a detailed analysis shows, within the limits of the perturbation theory used in this paper, the solution that takes into account both $g(z)$ and $w(z)$ dependence is the sum of a solution that assumes $g(z)$ and a constant $w$ with the solution that assumes $w(z)$ and a constant $g$. The former was obtained in Sec. V with $\tilde{\mathcal{E}}$ given by Eq. (67); the latter is obtained from Eq. (67) by simultaneous replacements $x \leftrightarrow y, x_{1} \leftrightarrow y_{1}$, and $g \leftrightarrow w$.

The applicability condition [Eq. (3)] can equivalently be written as $\left|a^{\prime}\right| \ll 1$ for the axisymmetric geometry and $\left|g^{\prime}\right| \ll 1$ for the rectangular one. Note also that, although we talked about a smooth transition from one pipe to another, it does not mean that the derivatives of the functions $a(z)$ and $g(z)$ should be continuous - jumps in these derivatives are allowed as long as the derivatives themselves are small. A typical situation in practice is when a transition is only piecewise smooth but all the angles are small. Our results are still applicable to this case, although they are less accurate than when the derivatives are continuous. A detailed study of the effect of discontinuous $a^{\prime}(z)$ with evaluation of correction terms is carried out in Ref. [11].

\section{ACKNOWLEDGMENTS}

I would like to thank B. Podobedov and S. Krinsky who pointed out a possible error in a numerical coefficient of the result of Ref. [3]. I also appreciate their comments on the draft version of this paper. I thank K. Bane for the critical reading of the manuscript. This work was supported by Department of Energy Contract No. DE-AC02$76 \mathrm{SF} 00515$.

[1] K. Yokoya, CERN Technical Report No. SL/90-88 (AP), 1990.

[2] G. Stupakov, SLAC Report No. SLAC-PUB-7167, 1996.

[3] G. Stupakov, Part. Accel. 56, 83 (1996).

[4] B. Podobedov and S. Krinsky, Phys. Rev. ST Accel. Beams 10, 074402 (2007).

[5] S. Krinsky, Phys. Rev. ST Accel. Beams 8, 124403 (2005).

[6] R.L. Warnock, SLAC Report No. SLAC-PUB-6038, 1993.

[7] G. V. Stupakov, SLAC Report No. SLAC-PUB-8857, 2001.

[8] W. K. H. Panofsky and W. Wenzel, Rev. Sci. Instrum. 27, 967 (1956).

[9] A. Chao, S. Heifets, and B. Zotter, Phys. Rev. ST Accel. Beams 5, 111001 (2002).

[10] R. L. Gluckstern, J. van Zeijts, and B. Zotter, Phys. Rev. E 47, 656 (1993).

[11] B. Podobedov and S. Krinsky, Phys. Rev. ST Accel. Beams 9, 054401 (2006). 